\title{
Secure Transparent Mobile IP for Intelligent Transportation Systems
}

\author{
Ann-Tzung Cheng, Chun-Hsin Wu, Jan-Ming Ho and D.T. Lee \\ Institute of Information Science \\ Academia Sinica, Taipei, Taiwan \\ \{jahorng, wuch, hoho,dtlee\}@iis.sinica.edu.tw
}

\begin{abstract}
In this paper we discuss the issues of wireless mobile networks for intelligent transportation systems (ITS) and examine several available technologies. Furthermore, we propose a design of secure transparent mobile IP to provide broadband ITS services over wireless LAN that improves upon security, transparency and handoff of mobile IP. The proposed design adopts a tunnel-in-tunnel mechanism for secure mobile network systems. It includes a direct registration method to support transparent registration and fast handoff, and a $V P N$-based access control to provide secure user roaming and traffic protection. Single sign-on is also developed to keep authenticated VPN sessions and mobile nodes connected even the attachment to Internet has changed. The protocol analysis and the preliminary experiment show that our design not only supports transparency and security for mobile IP, but it can also significantly reduce both the latency and the number of control messages incurred by handoff.
\end{abstract}

Keywords: mobile IP, WLAN, VPN, tunnel-in-tunnel, single sign-on.

\section{Introduction}

With the rapid progress in the development of computer and communication technologies, more and more intelligent transportation systems (ITS) are being developed to provide services through Internet, such as ITWS [1], Traffic-Channel [2] and Bus-Tracking [3]. For a cost-effective and efficient solution for ITS services over the Internet, we believe the following requirements of wireless access networks should be met: low cost, high mobility, large spatial capacity and open secure bidirectional transmission. Large spatial capacity will allow more users to access ITS services simultaneously in a dense population environment. The open features of the Internet will allow the ITS services to be provided by many different operators or providers, and requested by various users using common on-board units or wireless devices. As ITS services become more widely accessible through open mobile Internet, security and transparency will become more important.

There are many wireless access networks available for ITS applications to date. They can be simply classified into two groups: wide-area and short-range communication systems. The wide-area communication systems such as GSM and PACS are mainly used for emergency, broadcasting, narrowband, and high-speed mobility communications. In a city of high population density, however, these wide-area communication systems are not effective for continuous multimedia services and bi-directional communication applications due to limited shared bandwidth or high cost.

On the other hand, the short-range (or local-area) communication systems such as Dedicated Short Range Communication (DSRC) [4] and Wireless LAN (WLAN) [5] usually have higher bandwidth, but their low mobility restricts their applications. DSRC supports bi-directional broadband transmission, but its performance and cost are not as competitive as other communication systems to provide ITS services over the Internet. So the development of DSRC is coming closer to WLAN. For example, ASTM E2213 DSRC standard [6] is an extension of IEEE 802.11a WLAN standard.

Recently WLAN such as $802.11 \mathrm{~b}$ Wi-Fi networks has proved its success to provide wireless Internet access for enterprises and public space. Compared with wide-area communication systems, WLAN usually has low cost but high spatial capacity. To provide ITS services to mobile users beyond local area, however, several problems with WLAN must be solved. First, handoff latency becomes a significant problem because WLAN has small cell size that would cause more frequent handoffs. Besides, user roaming and traffic protection will be the fundamental problems in the wireless environment. Finally, for the compatibility requirement for heterogeneous mobile devices, it is important that the design of IP mobility management is transparent to existing on-board equipments and hand-held devices.

Several protocols such as wired equivalent privacy (WEP) and 802.1x [7] have been developed to improve the security of WLAN. However, they still can not support secure WLAN access well. The virtual private network (VPN) is a mature technology that protects access to a home network by a nomadic user. There are many research results [8][9][10][11] that have integrated mobile IP[12] with VPN technology to pursue secure mobility. 
The methods in [8][9][10] had a drawback that they require re-establishment of VPN that produces large handoff latency. The method in [11] only focused on protecting user traffic by VPN technique, and lacked the design of user roaming. Besides, all of them need the devices to understand mobile IP such that they can't be fully transparent to existing mobile devices.

The goal of transparent mobile IP is to allow almost all existing mobile devices, like PDA, laptop and IPenabled handhelds, to acquire IP mobility services easily without having to support extra layer 3 mobility management protocols, including mobile IP [12]. There are three issues to support transparent mobile IP: stale address resolution protocol (ARP) cache, home agent (HA) identification resolution and access control mechanism. Giovanardi and Mazzini [13][14] proposed a new network entity named Home Agent Server (HAS) to store identification information of all mobile nodes (MN's) to solve the HA identification problem, and proposed using media access control (MAC) address to authenticate MN. There are two drawbacks associated with their design insofar as to meet the requirements of the ITS wireless mobile network. First, the HA identification resolution process requires extra control messages to be delivered, which will increase some handoff latency. Second, the simple MAC address access control is not secure enough for the application of ITS.

To develop the wireless mobile network for ITS Internet services, we propose a new transparent mobile VPN that makes the design of secure mobility fully transparent to heterogeneous mobile devices, and integrates VPN technique to provide secure user roaming and traffic protection. This transparent mobile IP design improves transparent mobile IP [13][14] to pursue fast handoff. We also develop a single sign-on technique that would speed up the re-authentication process and make minimum damage to user communication.

The rest of the paper is organized as follows: In Section 2 we present our design of transparent mobile VPN. In Section 3, we show some experiments by comparing several methods. Finally, we give some conclusion in Section 4.

\section{Design of transparent mobile VPN}

For fast handoff and security, we propose a direct registration method to speed up transparent mobile IP registration process and a VPN-based access control to provide user roaming and traffic protection. In this section, we describe the design of transparent mobile VPN in four parts: direct registration, tunneling design, VPN-based access control and single sign-on. The first two parts are fundamental in the design of IP mobility management. The part of VPN-based access control illustrates our design of user roaming by applying VPN technique. For the requirement of short range communication, fast handoff is a key issue, and we will describe it in single sign-on part.

\subsection{Direct registration}

In our design, we propose a method, called direct registration, that enables a foreign agent (FA) to reach an HA successfully without relying on HAS or delivering extra messages. When an MN visits a foreign network, the FA can learn the IP address of the MN by monitoring packets from the MN. Then the FA can send out a mobile IP registration request message to the IP address of the MN without having to resolve the HA IP address first. Through the IP routing, the registration message will be routed to the MN's home network in the same way as packets are sent from a correspondent node $(\mathrm{CN})$ to the MN. Then the HA can intercept the message by Proxy ARP and handle the registration request. Although the control message will be mixed in the data traffic to the $\mathrm{MN}$, but the HA can easily distinguish a control message from data packets sent from CNs, by reserving a specific port number, i.e. UDP 434. After verifying the validity of the request, the HA will reply to the originating FA with its real IP address. Then the HA IP address is automatically and efficiently resolved by the FA.

Through the design of direct registration, the method of maintaining mobile node information in base mobile IP need not be modified, and no extra control messages are required. Besides, scalability would become an issue in the design of HA identification. This is because the fast growth of MNs and operators is a problem with the design of a central database management system. With the HAS design, it would need a distributed solution similar to the architecture of DNS to solve the scalability problem. Thus, direct registration is more flexible and efficient.

\subsection{Tunneling design}

Tunneling design plays an important role in packet protection and IP mobility. We adopt a tunnel-in-tunnel mechanism for secure mobile network systems. In our proposal, mobile IP tunneling works as the outer tunnel, handled by the mobile agents which carry packets and support roaming for the MN. Because the IP address of the $\mathrm{MN}$ is not topologically valid in the foreign network, packets with the MN IP address should be encapsulated into an IP tunneling between the foreign agent and the home agent. In addition, the VPN tunneling, either IPSEC tunneling or Generic Routing Encapsulation (GRE) tunneling, operates inside the mobile IP tunnel to protect data packets. This inner tunnel is established between the home VPN server and the MN. It is mainly used for encrypting packets for the $\mathrm{MN}$ and protecting them from eavesdropping attacks so no third party nodes can decrypt or masquerade these protected packets, including the FA. 


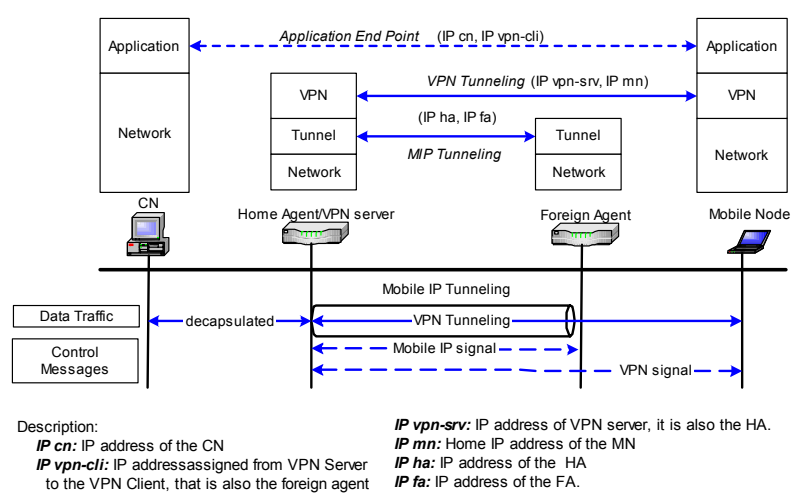

Figure 1. Tunneling in transparent mobile VPN

As shown in Figure 1, there are three IP addresses associated with an authenticated MN. The IP address, IPvpn-cli, is assigned by the home VPN server after the VPN connection has completed. All communications for upper layer applications that bind this IP address will be protected by the VPN tunneling. When the MN changes its visited FA, the VPN IP address will not be changed because the VPN connection will be kept by the outer mobile IP tunneling. And this also means that applications can stay alive and unaffected by handoff.

The second IP address is the home address IPmn. The home address is the network address of the MN used for accessing the foreign network, including establishing a VPN connection to its home. Similar to the VPN address, the home address should also keep unchanged when handoff occurs. Or the VPN session will be broken if the home address has changed.

Finally, the third address is so-called care-of address IPfa. This is also the IP address of the FA, which is used for mobile IP tunneling and indicates the current location of attachment to Internet for the MN. Through the care-of address, the HA can know which network the MN is in and where to forward traffic for the $\mathrm{MN}$. When an $\mathrm{MN}$ changes the visited FA, the care-of address will also be updated accordingly. The change is made by location update procedure like the mobile IP registration process between mobile agents.

Packets sent from the $\mathrm{MN}$ to a $\mathrm{CN}$ will first be encrypted into the VPN tunneling by its VPN client implementation and then encapsulated by the FA. By carrying into the mobile IP tunneling, these packets will be successfully routed to the HA through Internet. The end point of the mobile IP tunneling and the HA, which also functions as the VPN server, will do decapsulation before decryption. Then the HA can send out these packets originating from the $\mathrm{MN}$ to the target $\mathrm{CN}$. The $\mathrm{CN}$ will not be aware of these packets being sent from the $\mathrm{MN}$ directly

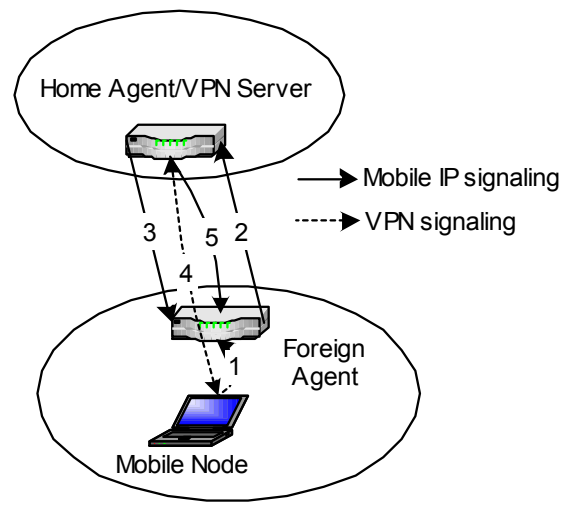

Figure 2. First login scenario

or through the HA and FA. Similarly, packets from the CN to the $\mathrm{MN}$ are processed in the reverse way.

\subsection{VPN-based access control}

When an unknown MN requests to access a network, it should set up a VPN connection successfully to do user authentication. As shown in Figure 2, the unknown user runs the first login procedure. After first login, the visitor initiates a VPN connection request to its home VPN server and then the first VPN signaling packet will trigger the FA to run mobile IP registration with the above direct registration mechanism. Messages 2 and 3 in Figure 2 are the mobile IP registration request and reply messages, respectively. All mobile IP control messages will be extended to carry the sender certificate and its signature to protect the integrity and get non-repudiation. After exchanging these mobile IP control messages, a mobile IP tunneling will be established between the two agents. At this time, the user has not been authenticated yet. Through the mobile IP tunneling, the MN can start to finish the VPN request and the user authentication will be done at this point. The user authentication algorithm can rely on the current methods used by VPN protocols, i.e. shared password, PKI-based and SIM-card. Finally, after user authentication is done by the VPN protocols, the HA will send the authentication result and authorization information to the visited FA via an authorization notification message, i.e., message 5 in Figure 2.

In this design, $\mathrm{MN}$ is directly authenticated by the HA following VPN protocols. It means that a user can use his home account to access foreign networks of other ISPs in the world; the user does not need any other roaming account to be authenticated by the foreign ISPs. In addition, the secure mobile IP tunnel is valid only after the HA authenticates the MN and the FA recognizes the VPN connection. The VPN tunnel is transparent to the FA, and the mobile IP tunnel is transparent to the MN. Though an FA does not authenticate a visitor itself, it can examine the authorization notification message sent from the HA with 


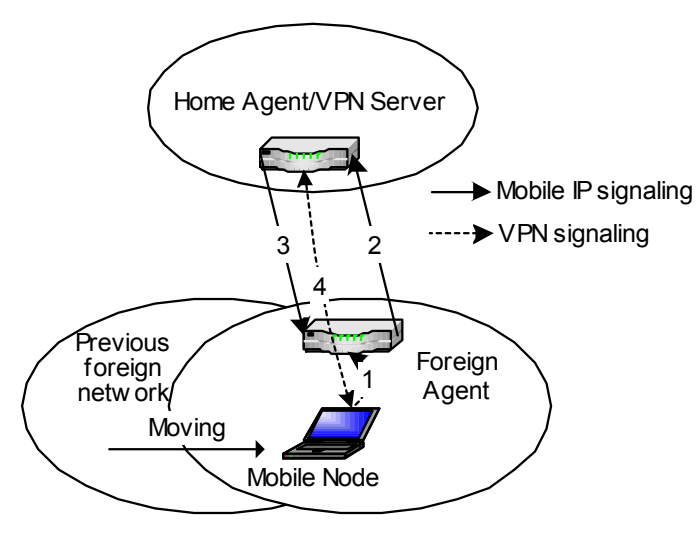

Figure 3. Single sign-on scenario

a valid signature to decide whether it would grant the visitor the access request. Besides, since the $\mathrm{MN}$ communicates with the HA using a VPN tunnel, it is almost impossible for a third-party node, including the previous and current FAs, to eavesdrop or tamper with the communication data. Hence the proposed approach can provide secure mobile access in an open wireless network by tunneling VPN packets inside a mobile IP tunnel.

\subsection{Single sign-on}

Because the time needed to set up a VPN connection is significant, it will degrade the communication quality if re-connection of VPN is needed whenever a handoff occurs. A feature, called single sign-on, is thus proposed. If a roaming user has logged in once, the user need not do extra login process after each handoff. The authenticated VPN session will keep in progress and allow the MN to be unaffected by the change of attachment to Internet.

In Figure 3, the MN has already initiated a VPN session to its home network in advance and has acquired authorization from the HA. After the MN moves to a new foreign network, once the VPN packet sent from the MN appears in the new foreign network, it will trigger the current FA to detect the arrival of the MN. Then the FA can send mobile IP registration request message to the MN home agent and append the VPN packets it captured in the control message as an authentication credential.

Most VPN protocols, such as IPSEC, give a sequence number on every protected packet to avoid replay attack. Besides, the VPN packets can only be decrypted by the correct session key held both on the home VPN server and the MN, so it is reasonable to take a VPN packet with correct sequence number as the credential for the MN. If the home VPN server can successfully decrypt an incoming VPN packet from a new FA and verify the correctness of the associated sequence number, the HA can trust that the MN is now moving to the requested foreign network. And then it can reply the message to the FA to establish a mobile IP tunneling for the MN. Again, the FA can decide to allow the access for the visitor by trusting the reply message from the HA.

\section{Analysis and experiment}

To evaluate the proposed secure transparent mobile IP network, we compared it with two previous works. We analyzed protocol behavior and measured handoff latency for each mechanism in the experiment.

\subsection{Experiment environment}

In the experiment, we constructed two foreign networks and one home network with the VPN server and RADIUS server. The MN moved from one foreign network to the other. All network nodes had synchronized network time by the NTP server. PPTP was chosen as the VPN protocol. We also set up two wireless-monitors that continuously listen to the wireless channels of the two access points. "Ethereal" was used to capture packets on all experiment nodes. The "Dynamics" project [15], a base mobile IP implementation developed by HUT, was used to run for mobile IP experiments. "NistNet" [16] emulator running on Linux was used to emulate the round trip time (RTT) between networks over Internet. We emulated the RTT between the home network and each foreign network to be $100 \mathrm{~ms}$ and the RTT between the $\mathrm{CN}$ and the home network to be $100 \mathrm{~ms}$. Flooding ping was run from the $\mathrm{MN}$ to the $\mathrm{CN}$ to help calculate the handoff latency.

In order to compare the difference between the authentication and mobility management in a handoff, we compared three mechanisms. The first one was mobile IP with $802.1 \mathrm{x}$ authentication. Because the layer 3 handoff latency of mobile IP mainly depends on the agent advertisement interval, we set it as 1 second that is the minimal setup value in "Dynamics". The $802.1 \mathrm{x}$ authentication was used for user roaming, and we chose EAP-TLS as the authentication method and WEP was not enabled.

The second one was mobile IP with VPN. The relevant setup of mobile IP was the same as that in first compared method. In order to compare the handoff latency contributed only by layer 3 handoff, the authentication method used was also the proposed VPN approach. In both methods, if a new agent advertisement is received by the $\mathrm{MN}$, it will cause a handoff immediately in this setup.

Finally, the third one is the proposed transparent mobile VPN. The certificates of agents were stored in each mobile agent in advance, so there wouldn't be extra messages delivered to exchange agent certificates in the registration process in all three compared methods. 


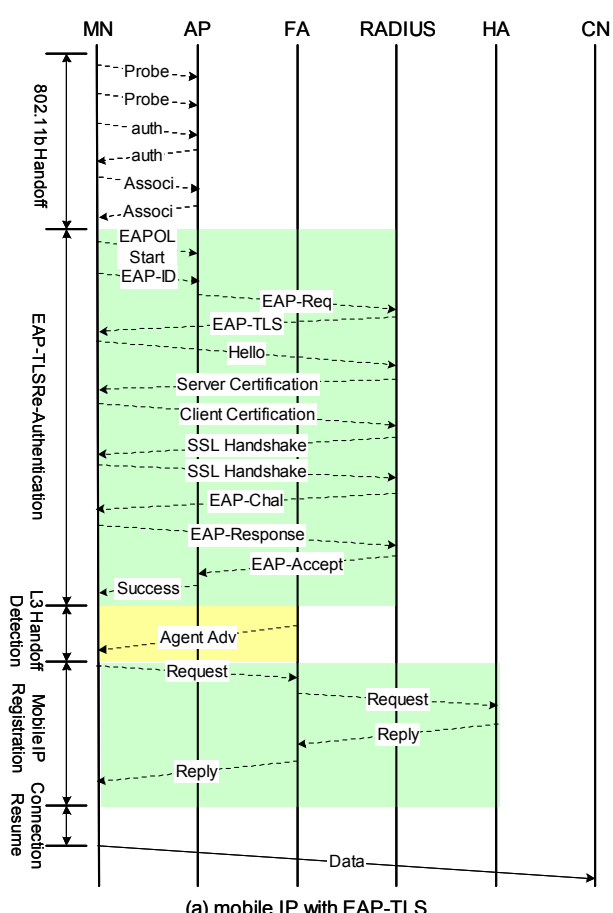

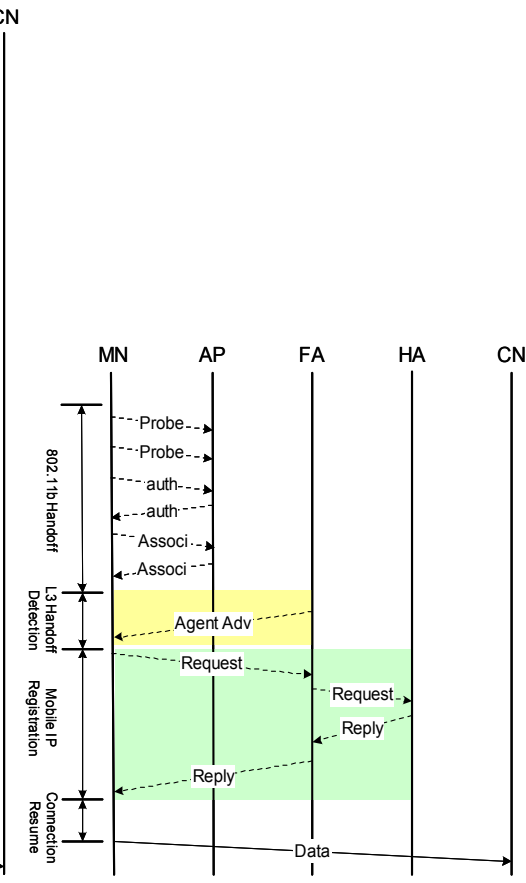

(b) mobile IP with VPN/SSO

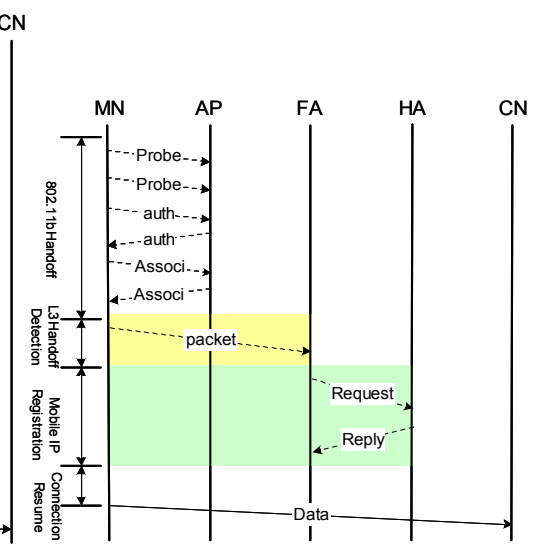

(c) transparent mobile VPN

Figure 4. Handoff details in different mechanisms

\subsection{Protocol analysis}

The handoff details in the three compared methods are shown in Figure 4. The process of layer 2 handoff is the same in three compared methods, so we will focus on the handoff latency incurred in authentication and layer 3. There are 5 parts in a handoff procedure. The first part is layer 2 handoff that relies on the underlying wireless technique. In the experiment, the Orinoco $802.11 \mathrm{~b}$ adapter had a good performance of which the average handoff latency is $88 \mathrm{~ms}$ in a total of 12 experiments. The second part is the re-authentication process. Without the design of single sign-on, the MN will need to do user authentication after each handoff. The third part is layer 3 handoff detection process used for the FA or the $\mathrm{MN}$ to detect the layer 3 handoff. In mobile IP, the handoff detection time mainly depends on the agent advertisement interval because the broadcasting advertisement can let the MN find the new foreign network and decide to do handoff. In transparent mobile IP, the handoff detection is made by any packet from the $\mathrm{MN}$ that appears in the foreign network. Therefore, the time is a variable and depends on the behavior of the MN. The fourth part is mobile IP registration process. The final part is the time for the first packet to be successfully transmitted to the $\mathrm{CN}$ after handoff is completed. The time is also a variable and depends on the behavior of the MN.

\subsection{Experiment results}

In Figure 5(a), the total handoff latency except layer 2 handoff latency is shown. Mobile IP with EAP-TLS had largest handoff latency that was averaged 2.32 second. Obviously it was mainly contributed by the reauthentication process that was averaged 1.58 second. The second largest latency with an average of 0.68 second was produced in mobile IP with VPN. Because the VPN single sign-on was used, no re-login was performed in the second compared method. So, the main contribution to the handoff latency was produced by mobile IP handoff detection mechanism with an average of 0.54 second. Theoretically, the handoff detection time is around half of the agent advertisement interval while the eager switch is enabled. The handoff latency in transparent mobile VPN had the smallest time of 0.16 second. The most difference between mobile IP and transparent mobile VPN was the layer 3 handoff detection. In transparent mobile IP, the average time was only 0.02 second. This was because flooding ping would send an ICMP request around each $15 \mathrm{~ms}$ that can speed up the time for the FA to detect the arrival of the MN.

Figure 5(b) shows the total number of control messages for mobile IP and authentication transmitted during a handoff procedure. Although it was obvious that mobile IP with EAP-TLS had the most control messages overhead, the most important was that the transmission path affected the cost of control message delivery. This is because the round trip time has large variation in LAN and WAN. It is straightforward that the total handoff latency of mobile IP with EAP-TLS was mainly contributed by the message delivered across WAN with RTT being $100 \mathrm{~ms}$ in our experiment. So reducing the number of messages delivered across WAN is more important than that across 

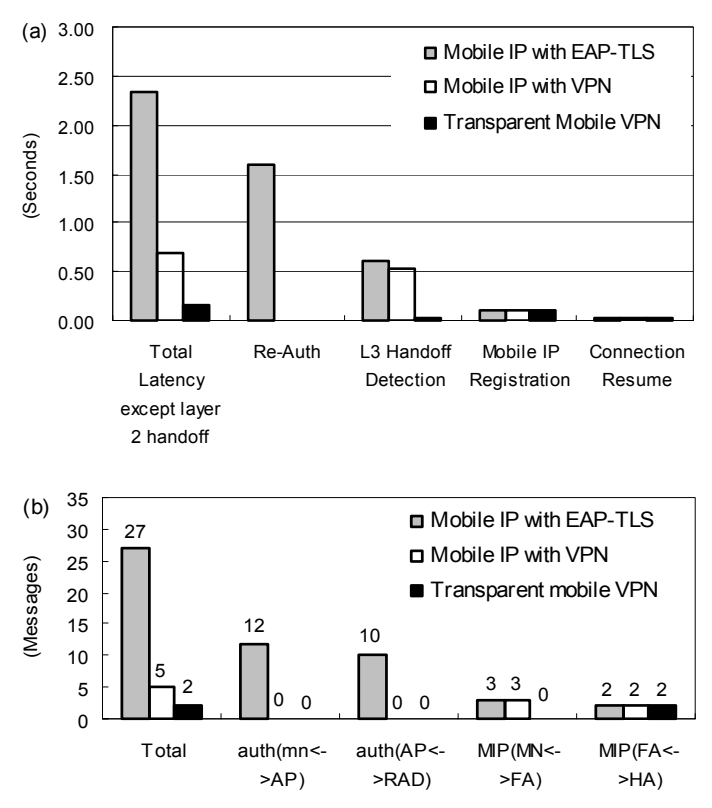

Figure 5. Experiment results : (a) handoff latency; (b) number of control message delivery

LAN. This is also a key contribution of our design of single sign-on.

\section{Conclusions}

In this paper we have proposed several novel ideas such as direct registration, VPN tunnel inside mobile IP tunnel, VPN-based access control and single sign-on to provide secure transparent access using enhanced mobile IP. The experiment shows that the proposed design can reduce handoff latency significantly. It also demonstrates that it is feasible to use short-range communication systems such as WLAN to support mobile access for broadband ITS applications.

\section{References}

[1] Chun-Hsin Wu, Da-Chun Su, Justin Chang, ChiaChen Wei, Jan-Ming Ho , Kwei-Jay Lin, and D. T. Lee, "An advanced traveler information system with emerging network technologies", Proc. 6th Asia-Pacific Intelligent Transportation Systems Forum, October 2003.

[2] ITS Research Program, University of Washington, "Traffic

http://www.its.washington.edu/trafchan/

[3] ITS Research Program, University of Washington, "My bus", http://www.mybus.org/

[4] American Society for Testing and Materials (ASTM), "Standard specification for Dedicated Short Range
Communication (DSRC) physical layer using microwave in the 902-928 MHz band”, ASTM E2158-01, April 1999.

[5] IEEE Std 802.11a-1999, "Information technology telecommunications and information exchange between systems - local and metropolitan area networks - specific requirements - Part 11: WLAN MAC and PHY specification", September 1999.

[6] American Society for Testing and Materials (ASTM), "Standard specification for telecommunications and information exchange between roadside and vehicle systems - $5 \mathrm{GHz}$ band Dedicated Short Range Communications (DSRC) Medium Access Control (MAC) and Physical Layer (PHY) specifications", ASTM E221302e1, October 2002.

[7] IEEE Std 802.1X, "Port-based network access control", 2001.

[8] T. Braun, and M. Danzeisen, "Secure mobile IP communication", Proc. 26th Annual IEEE Conference on Local Computer Networks (LCN), 2001.

[9] T. Braun, and M. Danzeisen, "Access of mobile IP users to firewall protected VPNs", Workshop on Mobile Communication over Wireless LAN at Informatik 2001 (WLAN/GIWS), Vienna, September 26-29, 2001.

[10] R. Bhagavathula, N. Thanthry, and R. Pendse, "Mobile IP and virtual private networks", Proc. 56th IEEE Vehicular Technology Conference (VTC), September 2002.

[11] M. Barton, D. Atkins, J. Lee, D.Ritcherson, K. Tepe, and K.D. Wong. "Integration of IP mobility and security for secure wireless communications", Proc. IEEE International Conference on Communications (ICC), 2002.

[12] C. Perkins, "IP mobility support for IPv4", Internet RFC 3220, January 2002.

[13] A. Giovanardi, and G. Mazzini, "Transparent mobile IP: an approach and implementation", Proc. IEEE Global Telecommunications Conference (GLOBECOM), 1997.

[14] A. Giovanardi, and G. Mazzini, "Optimization routing and security features for transparent mobile IP", Proc. IEEE Global Telecommunications Conference (GLOBECOM), 1998.

[15] Dynamics group, Helsinki University of Technology, "Dynamics: HUT mobile IP", http://www.cs.hut.fi/Research/Dynamics/

[16] National Institute of Standards and Technology, "NistNet", http://snad.ncsl.nist.gov/itg/nistnet/ 